\title{
Optimization of key parameters in the proton exchange membrane fuel cell
}

\author{
Hung-Hsiang Lin ${ }^{\mathrm{a}}$, Chin-Hsiang Cheng ${ }^{\mathrm{a}, *}$, Chyi-Yeou Soong ${ }^{\mathrm{b}}$, Falin Chen ${ }^{\mathrm{c}}$, Wei-Mon Yan ${ }^{\mathrm{d}}$ \\ a Department of Mechanical Engineering, Tatung University, 40 Chungshan N. Road, Sec. 3, Taipei 10451, Taiwan, ROC \\ ${ }^{\mathrm{b}}$ Department of Aerospace and Systems Engineering, Feng Chia University, Taichung 40724, Taiwan, ROC \\ ${ }^{\mathrm{c}}$ Institute of Applied Mechanics, National Taiwan University, Taipei 107, Taiwan, ROC \\ ${ }^{\mathrm{d}}$ Department of Mechatronic Engineering, Huafan University, Taipei 223, Taiwan, ROC
}

Received 2 May 2006; received in revised form 19 June 2006; accepted 19 June 2006

Available online 1 August 2006

\begin{abstract}
The present study is focused on the optimization of the proton exchange membrane fuel cell (PEMFC). A simplified conjugate-gradient method (SCGM) is employed to seek the optimal combination of the design parameters, including the channel width ratio $(\lambda)$, the porosity of GDL ( $\varepsilon_{\mathrm{GDL}}$ ), and the porosity of the catalyst layer $\left(\varepsilon_{\text {Cat }}\right)$. In this report, the concept of the method is described in detail and the results of the optimization process are discussed. The results show that the present approach is capable of providing an optimal design under specific operation conditions.
\end{abstract}

(C) 2006 Elsevier B.V. All rights reserved.

Keywords: PEM fuel cell; Optimization; Channel width ratio; Porosity; Model

\section{Introduction}

In the past several decades, fossil fuels have caused environmental pollution problems, such as the greenhouse effect and acid rain. Due to the increasing rate of consumption of global petroleum, finding other more efficient and cleaner substitutive power generation systems has become an issue of great concern. Among the possible alternative power generation systems, proton exchange membrane (PEM) fuel cells have received much attention from energy-related researchers and industries worldwide. Since in PEM fuel cells electric power is generated by directly transforming the chemical fuel energy into electricity in electrochemical reactions, these devices exhibit a higher efficiency compared to traditional power generators. Also, without ignition and combustion during electricity generation, PEM fuel cells are free of severe pollution and noise. In addition, PEM fuel cells do not have the flaws of corrosion of the mechanical elements and material problems associated with the working medium. Recently, PEM fuel cell technology has been developed more actively than ever in the automobile and $3 \mathrm{C}$ (computer, communication, consumer) electronics industries. The commercialisation of PEM fuel cells into mass production can be

\footnotetext{
* Corresponding author. Tel.: +886225925252 3410; fax: +8862 25997142. E-mail address: cheng@ttu.edu.tw (C.-H. Cheng).
}

accelerated. However, more efforts are still needed to explore the physical characteristic features as well as to improve the performance of PEM fuel cells in the coming years.

A number of reports have been presented in the past several decades; however, only those closely related to the present study are mentioned in the following.

Hental et al. [1] considered the effects of carbon plate calcinations at high temperature on the PEMFC efficiency. The authors claimed that, with a flow channel width of $1 \mathrm{~mm}$ and the thickness of the collector ribs of around $2 \mathrm{~mm}$, the performance of their fuel cell was obviously elevated.

Hontañón et al. [2] used CFD software to study the effects of GDL permeability and the ratio of the flow channel to the rib widths on the PEMFC performance. The results showed that a decrease in the permeability of the gas diffusion layer (GDL) reduces cell performance. It was also found that the influence of the GDL permeability on the cell performance is stronger than that of the flow channel width ratio.

Yoon et al. [3] similarly studied the influences of the flow channel width on the PEMFC efficiency. Their research showed that the gas diffusion effect plays a more important role than the electric conductivity effect. As the flow channel width is kept constant, the performance of fuel cell is higher with a smaller rib width.

Gurau et al. [4] developed a two-dimensional numerical model to study the changes in the gas concentration and 


\begin{tabular}{|c|c|}
\hline \multicolumn{2}{|c|}{ Nomenclature } \\
\hline$D$ & mass diffusivity $\left(\mathrm{m}^{2} \mathrm{~s}^{-1}\right)$ \\
\hline$F$ & Faraday constant $\left(96,487 \mathrm{C} \mathrm{mol}^{-1}\right)$ \\
\hline$I$ & current density $\left(\mathrm{A} \mathrm{m}^{-2}\right)$ \\
\hline$j$ & transfer current density $\left(\mathrm{A} \mathrm{m}^{-3}\right)$ \\
\hline$J$ & objective function \\
\hline$l_{\mathrm{b}}$ & base width of the flow distributor (m) \\
\hline$l_{\mathrm{c}}$ & gas channel width $(\mathrm{m})$ \\
\hline$l_{\mathrm{S}}$ & shoulder width (m) \\
\hline$M_{\mathrm{O}_{2}}$ & molar mass of oxygen $\left(32.0 \mathrm{~kg} \mathrm{kmol}^{-1}\right)$ \\
\hline$n$ & iteration step \\
\hline$n_{\mathrm{e}}$ & number of electrons \\
\hline$P$ & power output per unit area $\left(\mathrm{W} \mathrm{m}^{-2}\right)$ \\
\hline$R$ & universal gas constant $\left(8.314 \mathrm{~J}(\mathrm{~mol} \mathrm{~K})^{-1}\right)$ \\
\hline$t_{\mathrm{GDL}}$ & thickness of gas diffusion layer (m) \\
\hline$t_{\text {Cat }}$ & thickness of catalyst layer (m) \\
\hline$T$ & temperature $(\mathrm{K})$ \\
\hline$V$ & potential \\
\hline \multicolumn{2}{|c|}{ Greek letters } \\
\hline$\alpha$ & transfer coefficient for the reaction \\
\hline$\beta$ & step size \\
\hline$\gamma$ & conjugate-gradient coefficient \\
\hline$\varepsilon_{\text {Cat }}$ & porosity of catalyst layer \\
\hline$\varepsilon_{\mathrm{GDL}}$ & porosity of gas diffusion layer \\
\hline$\eta$ & over-potential (V) \\
\hline$\kappa$ & reaction rate constant \\
\hline$\lambda$ & channel width ratio, $l_{\mathrm{c}} / l_{\mathrm{b}}$ \\
\hline$\rho$ & density of oxygen $\left(\mathrm{kg} \mathrm{m}^{-3}\right)$ \\
\hline$\sigma$ & electric conductivity $\left(\Omega^{-1} \mathrm{~m}^{-1}\right)$ \\
\hline$\tau_{\text {Cat }}$ & tortuosity of catalyst layer \\
\hline$\tau_{\mathrm{GDL}}$ & tortuosity of gas diffusion layer \\
\hline$\phi$ & electric potential $(\mathrm{V})$ \\
\hline$\omega$ & oxygen mass fraction \\
\hline$\omega_{0}$ & oxygen mass fraction at inlet \\
\hline
\end{tabular}

electrochemical reaction rate by including the electrochemical kinetics of the catalyst layer and the convection-diffusion effect in the GDL. Results show that as the porosity of the GDL is increased, the entry velocity is elevated. They also found that the cell performance increases with the cell temperature.

Wang and Savinell [5] studied the effects of porosity, thickness, Pt-loading, CO poisoning of catalyst layer on the cell performance. In this report, it was observed that the catalyst layer porosity of an efficient fuel cell is between 0.4 and 0.7 .

Yan et al. [6] studied the influence of flow channel geometry and GDL porosity on gas transport. Results provided in this report showed that a larger flow channel width ratio or a higher GDL porosity leads to a better cell performance. They also pointed out that an increase in the number of flow channels results in a more uniform current density distribution and thus a better cell performance.

Zhou and Liu [7] proposed a three-dimensional fuel cell model to study the influence of $\mathrm{CO}$ toxins and the variation of hydrogen concentration in anode. Authors claimed that some phenomena, which were not found by using the one- and twodimensional models, are worthy of investigation by the threedimensional model. They also carried out a parametric analysis of the effects of the entry velocity, the porosity of the GDL, and the channel width ratio on the cell performance. Based on this report, it was observed that the channel width ratio and the GDL porosity both have their own optimal values.

Kumar and Reddy [8] studied the improvement of the performance of the PEMFC based on optimization of the dimensions and shape of the bipolar plates. It was found that the optimal dimensions for the channel width, the land width and the channel depth were close to $1.5,0.5$ and $1.5 \mathrm{~mm}$, respectively.

Hsu's work [9] was concerned with the analysis of the transport phenomenon and the parametric optimization of a PEM fuel cell. This study was focused on the influence of conductivity, channel width ratio, and porosity and thickness of the GDL. A two-dimensional half-cell model is utilized in simulation of the transport phenomenon.

Ying et al. [10] investigated the effects of channel configuration on an air-breathing fuel cell's performance. The authors found that the best performance can be obtained in the cell with a $3 \mathrm{~mm}$ wide cathode channel and an open ratio of $75.9 \%$.

Lum and McGuirk [11] carried out a series of parametric studies of the effects of variation of the electrode thickness, shoulder width, degree of permeability and oxidant concentration, so as to provide a clearer understanding of how changes in these parameters affect the cell performance.

Berning and Djilali [12] presented the results of a parametric study based on a three-dimensional, non-isothermal model of a PEM fuel cell. The authors evaluated the influence of the channel width ratio and the thickness and porosity of the GDL.

From the above survey of related literature, it is noted that most of the studies were focused on numerical simulation of the transport phenomenon in a PEM fuel cell, or at most on the parametric study of the effects of physical variables. Only a few reports dealt with the optimization of a set of design parameters.

In practice, the task of optimization searches for a combination of the most appropriate values of a number of design parameters with a minimized objective function. Nowadays, several useful optimization methods are available, for example, the genetic algorithm [13], the conjugate-gradient method (CGM) [14], the Newton-Raphson method [15], the adaptive weighting input estimation method [16], etc. These methods are all applicable to minimize the prescribed objective functions. Among them, the CGM method, which stems from perturbation principles, transforms the optimal problem into three separate problems, namely, the direct problem, the sensitivity problem, and the adjoining problem. The solutions of the three problems give the updated conjugate-gradient direction as well as the step size towards convergence. Cheng and Chang [17,18] used the concept of the conjugate-gradient method to develop an inverse method for predicting the internal temperature distribution of PEM fuel cells. The results show that the outer surface temperature data can be used to predict the internal temperature distribution at the interface between the carbon plate and the MEA. 
Under these circumstances, in the present study, a multiparametric optimization for the PEMFC design is investigated. Among the possible influential parameters, the channel width ratio, and the porosities of the GDL and the catalyst layer are recognized as the influential factors that may affect the behaviour of proton exchange and electron conduction; therefore, these three parameters are selected as the design parameters to be optimized in this work. The simplified conjugate-gradient method (SCGM) proposed by Cheng and Chang [14] is employed herein as the optimization search scheme. This study is aimed at optimization of these three parameters so that the best performance of the PEMFC under some specified geometry and operating conditions can be obtained. However, it is noted that the present optimization approach is not limited to the present group of designed parameters. Where necessary, more designed parameters may be readily applied.

\section{Optimization method}

The present optimization method consists of two major numerical solutions: (1) the direct problem solver and (2) the simplified conjugate-gradient method (SCGM). The two solvers are briefly described as follows.

\subsection{Direct problem solver}

Fig. 1 shows a schematic of a two-dimensional half-cell model for the cathode side employed for simulation of the transport phenomena involved. From top to bottom, the model consists of the catalyst layer, the gas diffusion layer, the flow channel, and the carbon plate which is developed with the following simplifications and assumptions:
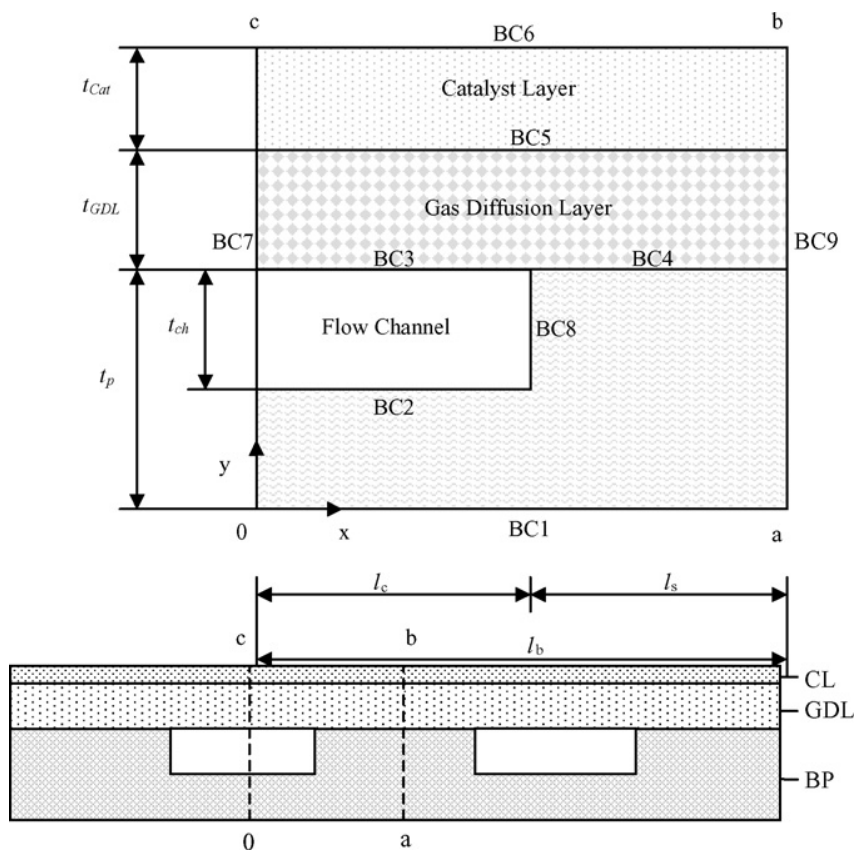

Fig. 1. Schematic of the two-dimensional simulation domain of half-cell PEMFC (cathode).
1. The fuel cell operates steadily.

2. The total pressure and temperature are uniform and constant in the solution domain.

3. The gases are assumed to be compressible ideal gases, fully saturated with water vapor.

4. The oxygen flux is zero at the interface between the catalyst layer and the membrane.

5. The gas transport from the flow channel to the catalyst layer is only by diffusion.

6. The gas diffusion layer and the catalyst layer are considered to be isotropic porous media.

7. Owing to high ohmic resistance of the membrane, the interface between the catalyst layer and the membrane is assumed to be insulated against electrons.

The channel width ratio $(\lambda)$ is defined by a ratio of the channel width $l_{\mathrm{c}}$ to the carbon plate width $l_{\mathrm{b}}$ as

$\lambda=\frac{l_{\mathrm{c}}}{l_{\mathrm{b}}}$

\subsubsection{Catalyst layer}

In the catalyst layer, the oxygen is consumed due to the electro-chemical reaction therefore, a two-dimensional transport equation for the oxygen mass fraction $\left(\omega_{\mathrm{Cat}}\right)$ in the catalyst layer can be expressed as

$$
\begin{gathered}
\frac{\partial}{\partial x}\left(\rho_{\mathrm{O}_{2}} D_{\mathrm{Cat}}^{\mathrm{eff}} \frac{\partial \omega_{\mathrm{Cat}}}{\partial x}\right)+\frac{\partial}{\partial y}\left(\rho_{\mathrm{O}_{2}} D_{\mathrm{Cat}}^{\mathrm{eff}} \frac{\partial \omega_{\mathrm{Cat}}}{\partial y}\right) \\
-\frac{j}{2 n_{\mathrm{e}} F} M_{\mathrm{O}_{2}}=0
\end{gathered}
$$

$D_{\mathrm{Cat}}^{\text {eff }}=\varepsilon_{\mathrm{Cat}}^{\tau_{\mathrm{Cat}}} D_{\mathrm{O}_{2}}$

where $\rho_{\mathrm{O}_{2}}$ stands for the oxygen density, $D_{\mathrm{O}_{2}}$ the oxygen diffusivity, $F$ the Faraday constant, $j$ the transfer current density, $n_{\mathrm{e}}$ the number of electrons in transfer current density relation, and $\varepsilon_{\text {Cat }}$ and $\tau_{\text {Cat }}$ represent the porosity and the tortuosity factor of the catalyst layer, respectively.

The cathode transfer current density can be expressed as

$j=n_{\mathrm{e}} F \kappa \omega \exp \left(\frac{\alpha n_{\mathrm{e}} F \eta}{R T}\right)$

where $\kappa$ is the reaction rate constant, $\alpha$ the transfer coefficient for the reaction, $\eta$ the over-potential, $R$ the universal gas constant, and $T$ is the cell temperature.

Due to the electro-chemical reactions activated in the catalyst layer, there exists a source term in the Poisson equation for the electric potential. That is

$$
\begin{aligned}
& \frac{\partial}{\partial x}\left(\sigma_{\text {Cat }}^{\text {eff }} \frac{\partial \phi_{\text {Cat }}}{\partial x}\right)+\frac{\partial}{\partial y}\left(\sigma_{\text {Cat }}^{\text {eff }} \frac{\partial \phi_{\text {Cat }}}{\partial y}\right)=j \\
& \sigma_{\text {Cat }}^{\text {eff }}=\left(1-\varepsilon_{\text {Cat }}\right)^{\tau_{\text {Cat }}} \sigma_{\text {Cat }}
\end{aligned}
$$

where $\sigma_{\text {Cat }}$ denotes the electric conductivity of the catalyst layer and $\phi_{\text {Cat }}$ the electric potential of the catalyst layer. 


\subsubsection{Gas diffusion layer (GDL)}

In the GDL, only the oxygen diffusion takes place without chemical reactions. The two-dimensional transport equation for the oxygen mass fraction $\omega_{\mathrm{GDL}}$ in the GDL can be expressed as

$\frac{\partial}{\partial x}\left(\rho_{\mathrm{O}_{2}} D_{\mathrm{GDL}}^{\mathrm{eff}} \frac{\partial \omega_{\mathrm{GDL}}}{\partial x}\right)+\frac{\partial}{\partial y}\left(\rho_{\mathrm{O}_{2}} D_{\mathrm{GDL}}^{\mathrm{eff}} \frac{\partial \omega_{\mathrm{GDL}}}{\partial y}\right)=0$

with

$D_{\mathrm{GDL}}^{\mathrm{eff}}=\varepsilon_{\mathrm{GDL}}^{\tau_{\mathrm{GDL}}} D_{\mathrm{O}_{2}}$

where $\varepsilon_{\mathrm{GDL}}$ and $\tau_{\mathrm{GDL}}$ are the porosity and the tortuosity factor of the GDL, respectively.

Since in the GDL there is no electrochemical reaction taking place, the electric potential equation possesses no electric source term and is expressed as

$\frac{\partial}{\partial x}\left(\sigma_{\mathrm{GDL}}^{\tau_{\mathrm{GDL}}} \frac{\partial \phi_{\mathrm{GDL}}}{\partial x}\right)+\frac{\partial}{\partial y}\left(\sigma_{\mathrm{GDL}}^{\tau_{\mathrm{GDL}}} \frac{\partial \phi_{\mathrm{GDL}}}{\partial y}\right)=0$

$\sigma_{\mathrm{GDL}}^{\mathrm{eff}}=\left(1-\varepsilon_{\mathrm{GDL}}\right)^{\tau_{\mathrm{Cat}}} \sigma_{\mathrm{GDL}}$

where $\sigma_{\mathrm{GDL}}$ is the electric conductivity and $\phi_{\mathrm{GDL}}$ is the electric potential in the GDL.

\subsubsection{Carbon plate}

It is essential to investigate the effects of gas channel width on the transport phenomena of the cathode reactants and on the ohmic resistance of the whole module.

The carbon plate serves as a conductor for the electric current. The electric potential equation in the carbon plate can be expressed as

$\frac{\partial}{\partial x}\left(\sigma_{\mathrm{p}} \frac{\partial \phi_{\mathrm{p}}}{\partial x}\right)+\frac{\partial}{\partial y}\left(\sigma_{\mathrm{p}} \frac{\partial \phi_{\mathrm{p}}}{\partial y}\right)=0$

where $\sigma_{\mathrm{p}}$ is the electric conductivity and $\phi_{\mathrm{p}}$ is the electric potential in the carbon plate.

\subsubsection{Boundary conditions}

The physical conditions at the boundaries numbered B1 to B9 in Fig. 1 are described as follows:

The boundary conditions for oxygen mass fraction $(\omega)$ are

- Catalyst layer:

$\frac{\partial \omega_{\mathrm{Cat}}}{\partial y}=0 \quad$ at boundary BC6;

$\omega_{\mathrm{Cat}}=\omega_{\mathrm{GDL}} \quad$ at boundary $\mathrm{BC} 5$;

$\frac{\partial \omega_{\mathrm{Cat}}}{\partial x}=0$ at boundaries BC7 and BC9

- GDL:

$D_{\mathrm{GDL}}^{\mathrm{eff}} \frac{\partial \omega_{\mathrm{GDL}}}{\partial y}=D_{\mathrm{Cat}}^{\mathrm{eff}} \frac{\partial \omega_{\mathrm{Cat}}}{\partial y} \quad$ at boundary BC5;

$\omega_{\mathrm{GDL}}=\omega_{0} \quad$ at boundary $\mathrm{B} 3$; $\frac{\partial \omega_{\mathrm{GDL}}}{\partial y}=0 \quad$ at boundaries BC4;

$\frac{\partial \omega_{\mathrm{GDL}}}{\partial x}=0$ at boundaries $\mathrm{BC} 7$ and $\mathrm{BC} 9$

The boundary conditions for electric potential $(\phi)$ are:

- Catalyst layer:

$\frac{\partial \phi_{\text {Cat }}}{\partial y}=0 \quad$ at boundary BC6;

$\phi_{\mathrm{Cat}}=\phi_{\mathrm{GDL}} \quad$ at boundary BC5;

$\frac{\partial \phi_{\text {Cat }}}{\partial x}=0 \quad$ at boundaries BC7 and BC9

- GDL:

$\sigma_{\mathrm{GDL}}^{\mathrm{eff}} \frac{\partial \phi_{\mathrm{GDL}}}{\partial y}=\sigma_{\mathrm{Cat}}^{\text {eff }} \frac{\partial \phi_{\mathrm{Cat}}}{\partial y} \quad$ at boundary BC5;

$\frac{\partial \phi_{\mathrm{GDL}}}{\partial y}=0 \quad$ at boundary BC3;

$\phi_{\mathrm{GDL}}=\phi_{\mathrm{p}} \quad$ at boundary $\mathrm{BC} 4$;

$\frac{\partial \phi_{\mathrm{GDL}}}{\partial x}=0 \quad$ at boundaries BC7 and BC9

- Carbon plate:

$\phi_{\mathrm{p}}=0 \quad$ at boundary $\mathrm{BC} 1$

$\sigma_{\mathrm{p}} \frac{\partial \phi_{\mathrm{p}}}{\partial y}=\sigma_{\mathrm{GDL}}^{\mathrm{eff}} \frac{\partial \phi_{\mathrm{GDL}}}{\partial y} \quad$ at boundary BC4;

$\frac{\partial \phi_{\mathrm{p}}}{\partial x}=0$ at boundaries BC7, BC8, and BC9;

$\frac{\partial \phi_{\mathrm{p}}}{\partial y}=0 \quad$ at boundary BC2

\subsubsection{Operation potential}

With the solutions for the electric potential distribution yielded using the above model, the cell potential $(V)$ can be calculated by the following equation:

$V=V_{\mathrm{OC}}-\eta-\Delta \phi$

where $V_{\mathrm{OC}}$ denotes the ideal cell potential, $\eta$ is for the overpotential of the cathode, and

$\Delta \phi=\int_{0}^{l_{\mathrm{p}}} \frac{\left(\phi_{6}-\phi_{1}\right) \mathrm{d} x}{l_{\mathrm{p}}}$

denoting the average ohmic loss in terms of the difference between the electric potentials at boundaries B6 and B1, respectively. During the optimization, the cell voltage $V$ and cell current $I$ are varied accompanying iterative changes in the design parameters.

It is noted that the present two-dimensional model is rather straightforward and not able to include the effects of heat and liquid water accumulation. A more powerful model will certainly be required. However, the simplicity of the model is acceptable, 
given that only the channel width ratio and the porosities of the GDL and the catalyst layer are taken into account and the optimization is emphasized rather than the complexity of the model.

\subsection{Simplified conjugate-gradient method (SCGM)}

In this study, the simplified conjugate-gradient method (SCGM) [10] is employed to optimize the values of the design parameters in search of optimal performance. The objective function in conjunction with the optimization process is defined in the following:

$J\left(A_{k}\right)=\frac{1}{P}, \quad k=1,2,3$

where $A_{k}(k=1,2$, and 3$)$ represent the channel width ratio $(\lambda)$, the porosity of the GDL ( $\left.\varepsilon_{\mathrm{GDL}}\right)$, and the porosity of the catalyst layer $\left(\varepsilon_{\text {Cat }}\right)$. The cell power output $(P)$ is expected to reach a maximum as the magnitude of the objective function $J$ is minimized. In the SCGM method, the values of the step size $\beta_{k}$ during the searching procedure are fixed. In this manner, the complexities in the mathematical manipulation and the constraint of the form of the objective function can be alleviated. The initial guess for each design variable is made first, and in the successive steps the conjugate-gradient coefficients and the searching directions are evaluated. The new design variables are continuously improved. When the objective function reaches a minimum, the goal of the optimization is reached and the searching procedure is then terminated.

The procedure for the iterative optimization process with the SCGM method is presented as follows:

(1) Make the initial guess for the designed variables $A_{k}$, assign the values to the step sizes $\beta_{k}$.

(2) Specify all boundary conditions, and then solve the direct problem.

(3) Calculate the objective function $J\left(A_{k}, k=1-3\right)$. If the convergence criterion is satisfied as the objective function reaches a minimum in the iterating process, the solution process is terminated. Otherwise, proceed to step (4).

(4) Calculate the gradient functions of the function $J$ :

$$
\frac{\partial J^{n}}{\partial A_{k}}=\frac{\Delta J^{n}}{\Delta A_{k}}, \quad k=1-3
$$

(5) Calculate the conjugate-gradient coefficients $\gamma_{k}^{n}(k=1-3)$ :

$$
\gamma_{k}^{n}=\left[\frac{\left(\partial J / \partial A_{k}\right)^{n}}{\left(\partial J / \partial A_{k}\right)^{n-1}}\right]^{2}, \quad k=1-3
$$

For the first step with $n=0, \gamma_{k}^{0}=0$.

(6) Calculate the searching directions $\xi_{k}^{n}(k=1-3)$ :

$$
\xi_{k}^{n+1}=\frac{\partial J^{n}}{\partial A_{k}}+\gamma_{k}^{n} \xi_{k}^{n}, \quad k=1-3
$$

(7) Update new design variables by

$$
A_{k}^{n+1}=A_{k}^{n}-\beta_{k} \xi_{k}^{n+1}, \quad k=1-3
$$

\begin{tabular}{|c|c|c|}
\hline Parameters & Notation & Value \\
\hline Temperature $(\mathrm{K})$ & $T$ & 333 \\
\hline Reaction rate constant $\left(\mathrm{mol}\left(\mathrm{m}^{3} \mathrm{~s}\right)^{-1}\right)$ & $\kappa$ & $1.5 \times 10^{-}$ \\
\hline Oxygen mass fraction at inlet & $\omega_{0}$ & 0.17 \\
\hline Gas channel width $(\mathrm{m})$ & $l_{\mathrm{c}}$ & $1.0 \times 10^{-}$ \\
\hline Channel shoulder width (m) & $l_{\mathrm{s}}$ & $1.0 \times 10^{-}$ \\
\hline Thickness of gas diffusion layer (m) & $t_{\mathrm{GDL}}$ & $5 \times 10^{-4}$ \\
\hline Thickness of catalyst layer (m) & $t_{\text {Cat }}$ & $5 \times 10^{-5}$ \\
\hline Porosity of GDL & $\varepsilon_{\mathrm{GDL}}$ & 0.5 \\
\hline Tortuosity of GDL & $\tau_{\mathrm{GDL}}$ & 1.5 \\
\hline Porosity of catalyst layer & $\varepsilon_{\text {Cat }}$ & 0.3 \\
\hline Tortuosity of catalyst layer & $\tau_{\text {Cat }}$ & 1.5 \\
\hline Faraday constant $\left(\mathrm{C} \mathrm{mol}^{-1}\right)$ & $F$ & 96487 \\
\hline Universal gas constant $\left(\mathrm{J}(\mathrm{mol} \mathrm{K})^{-1}\right)$ & $R$ & 8.314 \\
\hline Molar mass of oxygen $\left(\mathrm{kg} \mathrm{kmol}^{-1}\right)$ & $M_{\mathrm{O}_{2}}$ & 32.0 \\
\hline Number of electrons & $n_{\mathrm{e}}$ & 2 \\
\hline Transfer coefficient for reaction & $\alpha$ & 1 \\
\hline Electric conductivity of catalyst layer $\left(\Omega^{-1} \mathrm{~m}^{-1}\right)$ & $\sigma_{\text {Cat }}$ & 190 \\
\hline Electric conductivity of GDL $\left(\Omega^{-1} \mathrm{~m}^{-1}\right)$ & $\sigma_{\mathrm{GDL}}$ & 537 \\
\hline Electric conductivity in carbon plate $\left(\Omega^{-1} \mathrm{~m}^{-1}\right)$ & $\sigma_{\mathrm{p}}$ & 4000 \\
\hline
\end{tabular}

and then return to step (2).
Table 1

Variables used in the present analysis
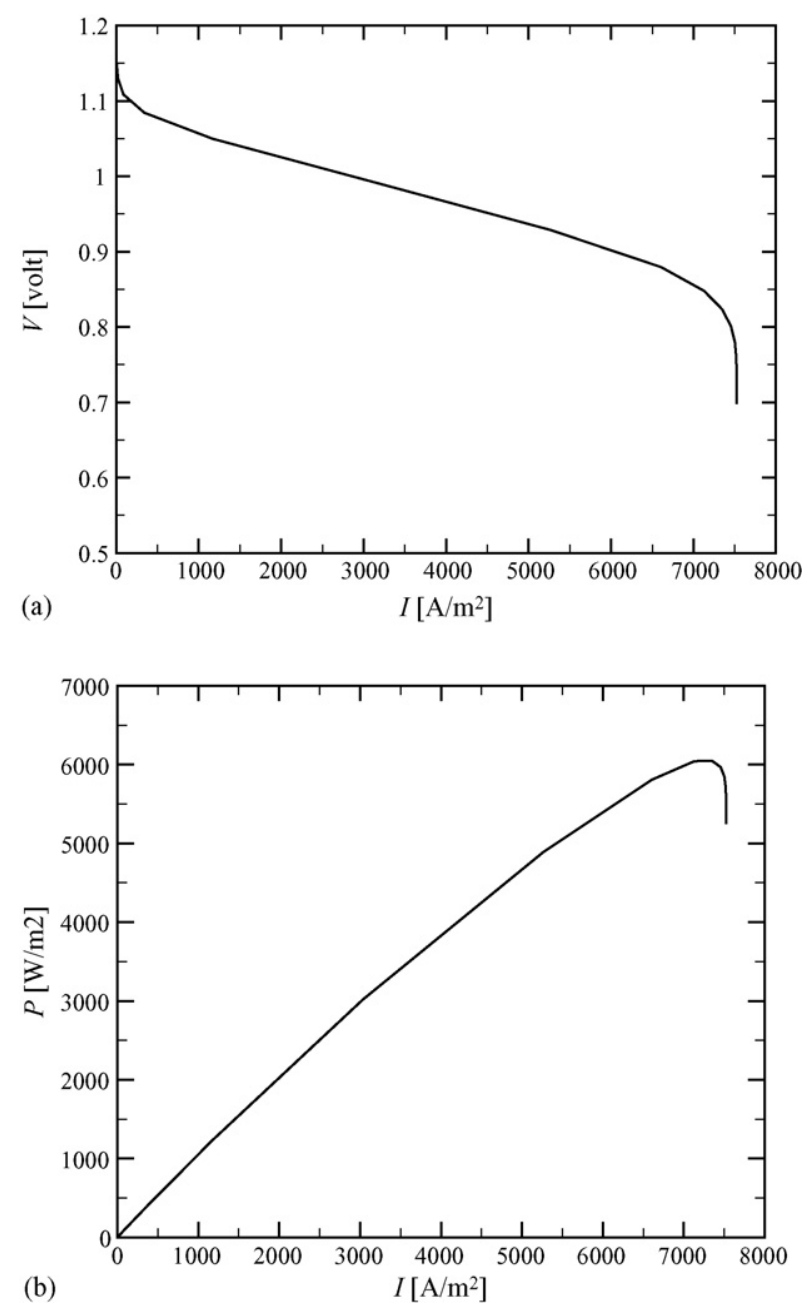

Fig. 2. A typical case at conditions of $\sigma_{\mathrm{Cat}}=190 \Omega^{-1} \mathrm{~m}^{-1}, \sigma_{\mathrm{GDL}}=537$ $\Omega^{-1} \mathrm{~m}^{-1}, \sigma_{\mathrm{p}}=4000 \Omega^{-1} \mathrm{~m}^{-1}, t_{\mathrm{GDL}}=5 \times 10^{-4} \mathrm{~m}, t_{\mathrm{Cat}}=5 \times 10^{-5} \mathrm{~m}, \lambda=0.5$, $\varepsilon_{\mathrm{GDL}}=0.5$, and $\varepsilon_{\mathrm{Cat}}=0.3$. (a) Polarization curve and (b) power curve of a PEM fuel cell. 


\section{Results and discussion}

\subsection{Preliminary study}

In the computation, the activated over-potential acts as an independent variable to replace the external loads. By doing so, the polarization diagram of the fuel cell can be determined. The power density of the fuel cell is the product of the potential $V$ and the current density $I$ as

$P=V I$

The model described in the preceding section is solved by a finite volume method with a uniformly distributed grid having 101 and 511 nodes in the $x$ - and $y$-directions, respectively. The values of the geometrical and physical variables considered in the present study are listed in Table 1.

Fig. 2 shows the polarization and the power curves for a typical case at the conditions of $\sigma_{\text {Cat }}=190 \Omega^{-1} \mathrm{~m}^{-1}$,
$\sigma_{\mathrm{GDL}}=537 \Omega^{-1} \mathrm{~m}^{-1}, \sigma_{\mathrm{p}}=4000 \Omega^{-1} \mathrm{~m}^{-1}, t_{\mathrm{GDL}}=5 \times 10^{-4} \mathrm{~m}$, $t_{\text {Cat }}=5 \times 10^{-5} \mathrm{~m}, \lambda=0.5, \varepsilon_{\mathrm{GDL}}=0.5$, and $\varepsilon_{\mathrm{Cat}}=0.3$. For a lower over-potential, the weaker electrochemical reaction leads to a lower power density. However, when the over-potential is increased, the reaction is stronger so that the power density reaches a maximum at $I=7400 \mathrm{~A} \mathrm{~m}^{-2}$.

Sensitivity analysis of the three optimized design parameters with respect to the cell voltage has been carried out and Fig. 3(a)-(c) are plotted to show the results. The cases shown in Fig. 3(a)-(c) are at $\sigma_{\mathrm{Cat}}=190 \Omega^{-1} \mathrm{~m}^{-1}$, $\sigma_{\mathrm{GDL}}=537 \Omega^{-1} \mathrm{~m}^{-1}, \sigma_{\mathrm{p}}=4000 \Omega^{-1} \mathrm{~m}^{-1}, t_{\mathrm{GDL}}=5 \times 10^{-4} \mathrm{~m}$, $t_{\text {Cat }}=5 \times 10^{-5} \mathrm{~m}$. Fig. 3(a) shows the effects of the channel width ratio $(\lambda)$ for $\varepsilon_{\mathrm{GDL}}=0.5$ and $\varepsilon_{\mathrm{Cat}}=0.3$, Fig. 3(b) shows the effects of porosity of GDL ( $\varepsilon_{\mathrm{GDL}}$ ) for $\lambda=0.5$ and $\varepsilon_{\mathrm{Cat}}=0.3$, and Fig. 3(c) conveys the effects of porosity of catalyst layer $\left(\varepsilon_{\mathrm{Cat}}\right)$ for $\lambda=0.5$ and $\varepsilon_{\mathrm{GDL}}=0.5$. It is found that in the ranges of parameters considered, the cell voltage is more sensitive to a change in the channel width ratio or the porosity of the GDL.
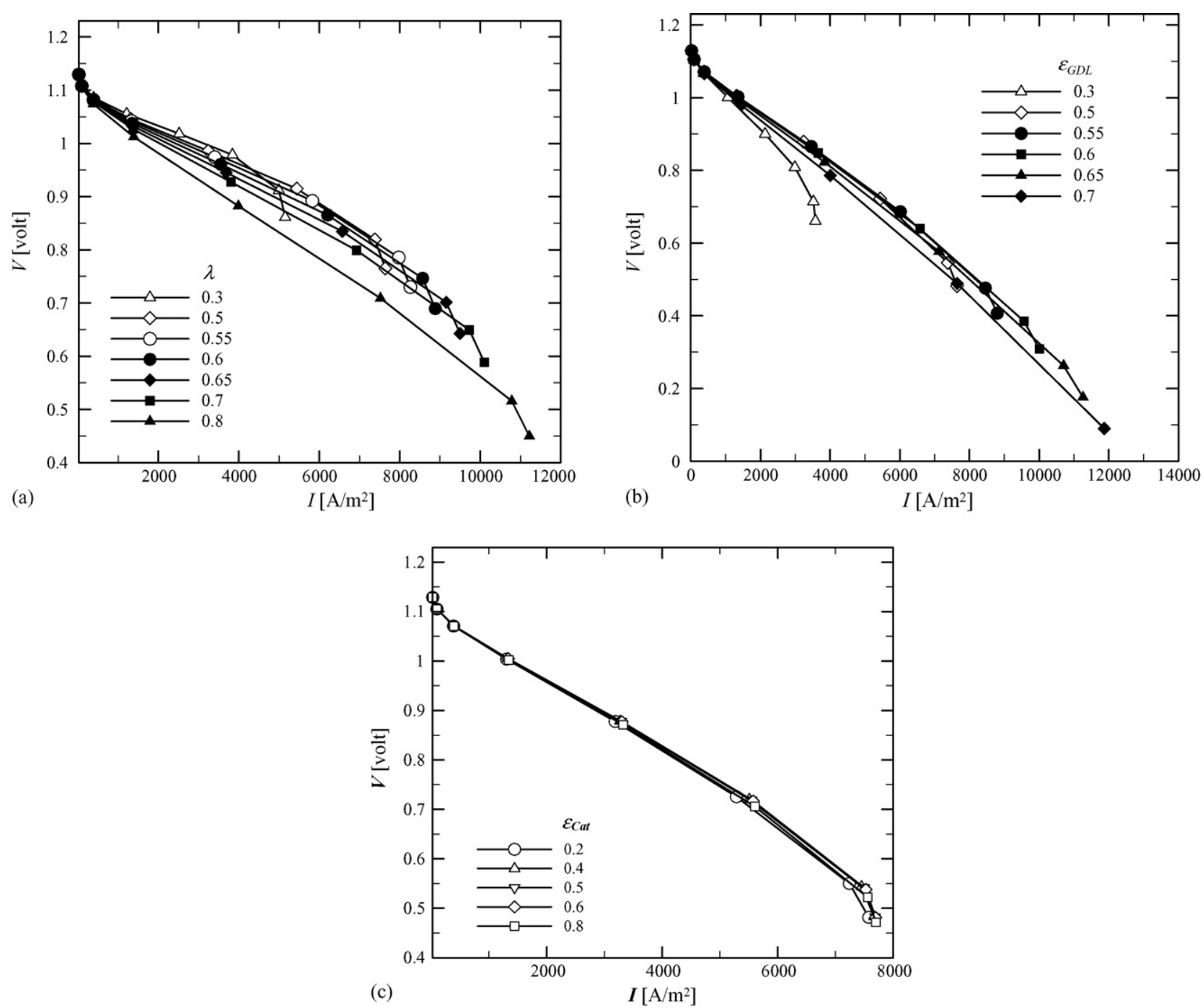

Fig. 3. Dependence of cell voltage on designed parameters at $\sigma_{\mathrm{Cat}}=190 \Omega^{-1} \mathrm{~m}^{-1}, \sigma_{\mathrm{GDL}}=537 \Omega^{-1} \mathrm{~m}^{-1}, \sigma_{\mathrm{p}}=4000 \Omega^{-1} \mathrm{~m}^{-1}, t_{\mathrm{GDL}}=5 \times 10^{-4} \mathrm{~m}, t_{\mathrm{Cat}}=5 \times 10^{-5} \mathrm{~m}$. (a) Effects of channel width ratio $(\lambda)$ for $\varepsilon_{\mathrm{GDL}}=0.5$ and $\varepsilon_{\mathrm{Cat}}=0.3$. (b) Effects of porosity of GDL $\left(\varepsilon_{\mathrm{GDL}}\right)$ for $\lambda=0.5$ and $\varepsilon_{\mathrm{Cat}}=0.3$. (c) Effects of porosity of catalyst layer $\left(\varepsilon_{\mathrm{Cat}}\right)$ for $\lambda=0.5$ and $\varepsilon_{\mathrm{GDL}}=0.5$. 
However, it is relatively less sensitive to the porosity of the catalyst layer.

Fig. 4 further shows the effects of the channel width ratio ( $\lambda$ ) on the performance of a PEM fuel cell at $\eta=0.25 \mathrm{~V}$, $t_{\mathrm{GDL}}=5 \times 10^{-4} \mathrm{~m}, t_{\mathrm{Cat}}=5 \times 10^{-5} \mathrm{~m}, \varepsilon_{\mathrm{GDL}}=0.5$, and $\varepsilon_{\mathrm{Cat}}=0.3$. It is observed that indeed the channel width ratio has a subtle influence on the cell performance. Larger channel width ratios may enhance the fuel gas transport as well as the chemical reaction in the catalyst layer; however, a larger channel width leads to smaller ribs and hence results in an increase in the ohmic impedance. With these two opposing effects, there must be an optimal value for the channel width ratio $(\lambda)$, at which the performance reaches a peak value. In Fig. 4, the results show that the largest power of $6572 \mathrm{~W} \mathrm{~m}^{-2}$ is obtained at the optimal channel width ratio of $\lambda=0.65$ under the conditions considered in this case.

Fig. 5 shows the effects of the GDL porosity $\left(\varepsilon_{\mathrm{GDL}}\right)$ on the performance of a PEM fuel cell at $\eta=0.25 \mathrm{~V}$. A higher GDL porosity implies a higher ohmic impedance and results in a reduction in the electric current. However, on the other hand, a higher GDL porosity also implies a stronger diffusion of reactant gas in the GDL. Fig. 5 shows that the optimal value of $\varepsilon_{\mathrm{GDL}}$ is 0.6 at $\eta=0.25 \mathrm{~V}, \varepsilon_{\text {Cat }}=0.3, t_{\mathrm{GDL}}=5 \times 10^{-4} \mathrm{~m}, t_{\mathrm{Cat}}=5 \times 10^{-5} \mathrm{~m}$, and $\lambda=0.5$. At the optimal value of $\varepsilon_{\mathrm{GDL}}$, the power density approaches $6843 \mathrm{~W} \mathrm{~m}^{-2}$.

The effects of the catalyst layer porosity $\left(\varepsilon_{\mathrm{Cat}}\right)$ on the performance of a PEM fuel cell at $\eta=0.25 \mathrm{~V}, \varepsilon_{\mathrm{GDL}}=0.5$, $t_{\mathrm{GDL}}=5 \times 10^{-4} \mathrm{~m}, t_{\mathrm{Cat}}=5 \times 10^{-5} \mathrm{~m}$, and $\lambda=0.5$ are plotted in Fig. 6. Except for the case at very low or very high porosity $\varepsilon_{\text {Cat }}$, the effects of the catalyst layer porosity are not as obvious as those of the GDL porosity. This is attributed to the fact that the catalyst layer is relatively thin, and hence the effects of $\varepsilon_{\text {Cat }}$ on the performance are limited. The variation of the power output is only less than $1 \%$ as the porosity $\varepsilon_{\mathrm{Cat}}$ is in the range of $0.3-0.8$. Even so, the optimal porosity of the catalyst layer $\varepsilon_{\mathrm{Cat}}$ is also obtained. For the case at $\eta=0.25 \mathrm{~V}, \varepsilon_{\mathrm{GDL}}=0.5$,

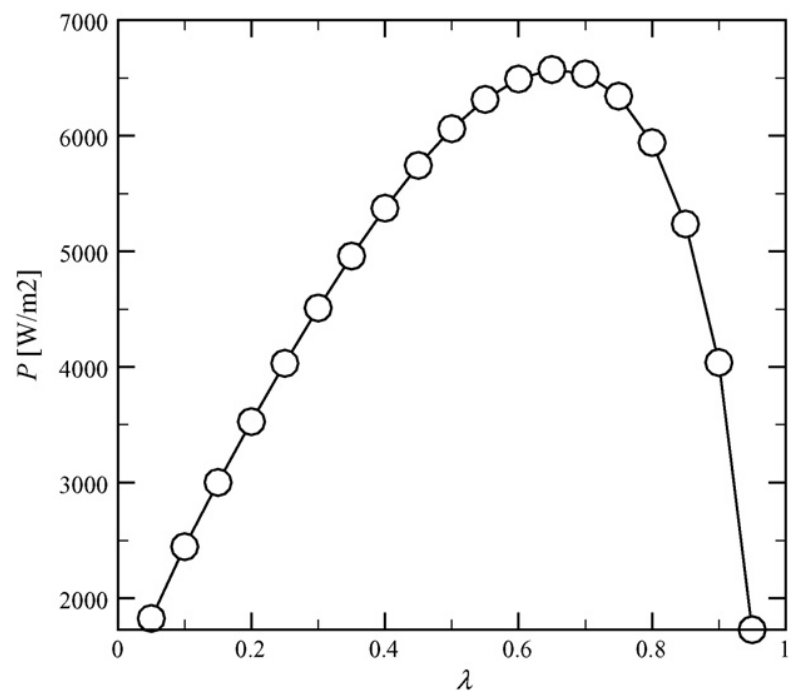

Fig. 4. Effects of channel width ratio $\lambda$ on performance of a PEM fuel cell at $\eta=0.25 \mathrm{~V}, t_{\mathrm{GDL}}=5 \times 10^{-4} \mathrm{~m}, t_{\mathrm{Cat}}=5 \times 10^{-5} \mathrm{~m}, \varepsilon_{\mathrm{GDL}}=0.5$, and $\varepsilon_{\mathrm{Cat}}=0.3$.

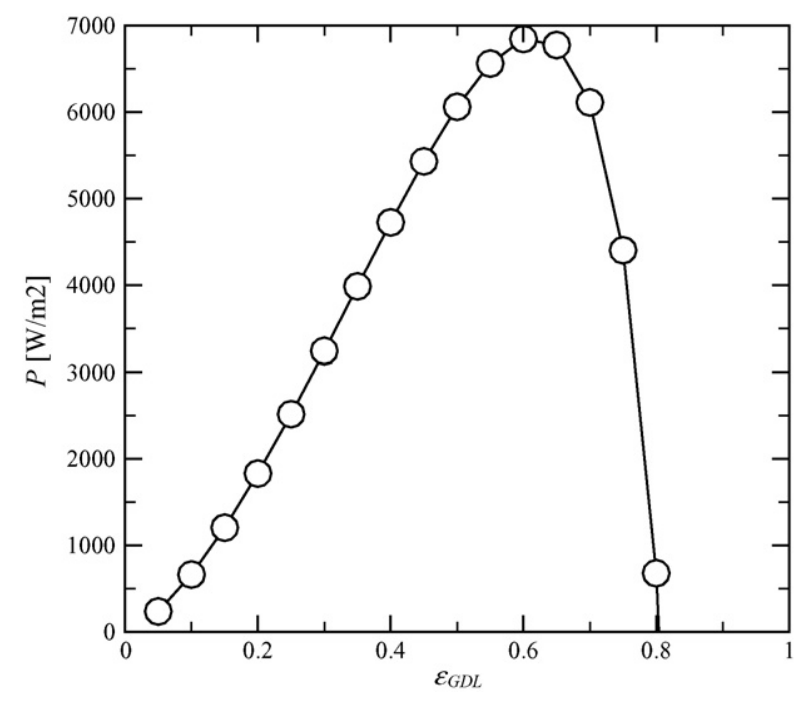

Fig. 5. Effects of GDL porosity $\varepsilon_{\mathrm{GDL}}$ on performance of a PEM fuel cell at $\eta=0.25 \mathrm{~V}, \varepsilon_{\mathrm{Cat}}=0.3, t_{\mathrm{GDL}}=5 \times 10^{-4} \mathrm{~m}, t_{\mathrm{Cat}}=5 \times 10^{-5} \mathrm{~m}$, and $\lambda=0.5$.

$t_{\mathrm{GDL}}=5 \times 10^{-4} \mathrm{~m}, t_{\mathrm{Cat}}=5 \times 10^{-5} \mathrm{~m}$, and $\lambda=0.5$, the optimal $\varepsilon_{\text {Cat }}$ is 0.5 and the corresponding power of $6099 \mathrm{~W} \mathrm{~m}^{-2}$ can be observed.

\subsection{Optimization of PEMFC}

In the parametric studies considered above, only one parameter is changed while others are fixed. In fact, multi-parameter optimization is more meaningful from the design viewpoint. Fig. 7 shows the trajectory of the multi-parameter optimization process approaching the optimal design. The optimal combination of $\lambda, \varepsilon_{\mathrm{GDL}}$, and $\varepsilon_{\mathrm{Cat}}$ are sought in the parameter space $\left(\lambda, \varepsilon_{\mathrm{GDL}}, \varepsilon_{\mathrm{Cat}}\right)$. The optimization process is started with the initial guess of $\lambda=0.65, \varepsilon_{\mathrm{GDL}}=0.5$, and $\varepsilon_{\mathrm{Cat}}=0.3$. It can be observed in Fig. 7 that the SCGM method effectively brings the searching process to approach an optimal state. In this case,

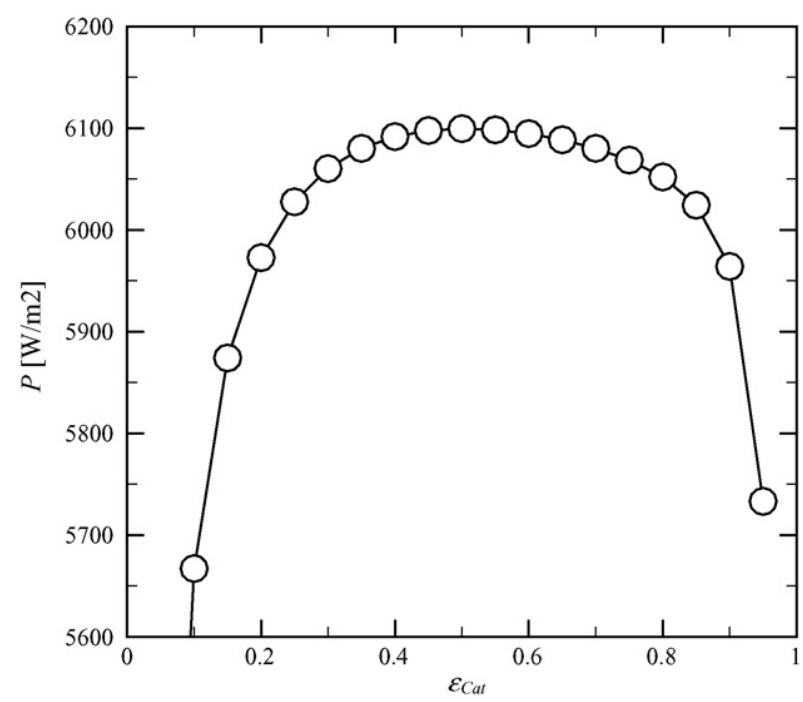

Fig. 6. Effects of catalyst layer porosity $\varepsilon_{\mathrm{Cat}}$ on performance of a PEM fuel cell at $\eta=0.25 \mathrm{~V}, \varepsilon_{\mathrm{GDL}}=0.5, t_{\mathrm{GDL}}=5 \times 10^{-4} \mathrm{~m}, t_{\mathrm{Cat}}=5 \times 10^{-5} \mathrm{~m}$, and $\lambda=0.5$. 


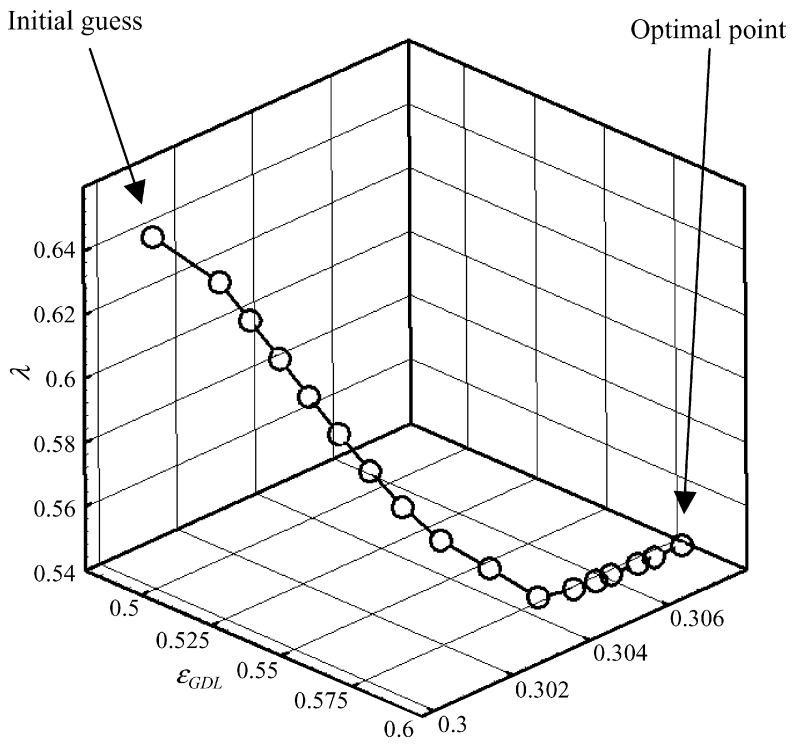

Fig. 7. Trajectory of the optimization process for a PEM fuel cell seeking an optimal set of designed parameters $\lambda, \varepsilon_{\mathrm{GDL}}$, and $\varepsilon_{\mathrm{Cat}}$.

it is found that the iteration converges after about 400 iterations. The computation time required is approximately $80 \mathrm{~h}$ on a personal computer with an AMD-3GMHz CPU. Based on the results shown in Fig. 7, it is found that at $\lambda=0.55$, $\varepsilon_{\mathrm{GDL}}=0.5913$ and $\varepsilon_{\mathrm{Cat}}=0.307$, the optimization performance can be generated with a maximum power of $6866.53 \mathrm{~W} \mathrm{~m}^{-2}$ at $\eta=0.25 \mathrm{~V}, t_{\mathrm{GDL}}=5 \times 10^{-4} \mathrm{~m}, t_{\mathrm{Cat}}=5 \times 10^{-5} \mathrm{~m}$. The variation of the power density during the optimization process is shown in Fig. 8. Note that the power density associated with the initial design is approximately $2500 \mathrm{~W} \mathrm{~m}^{-2}$. The power density then approaches its maximum value when the optimal parameters are obtained.

To check the robustness of the searching method used in the present work, Fig. 9 shows the convergence of the optimization process from three different initial guesses for the three parameters $\lambda, \varepsilon_{\mathrm{GDL}}$, and $\varepsilon_{\mathrm{Cat}}$, at $\eta=0.25 \mathrm{~V}$. It can be observed in Fig. 9

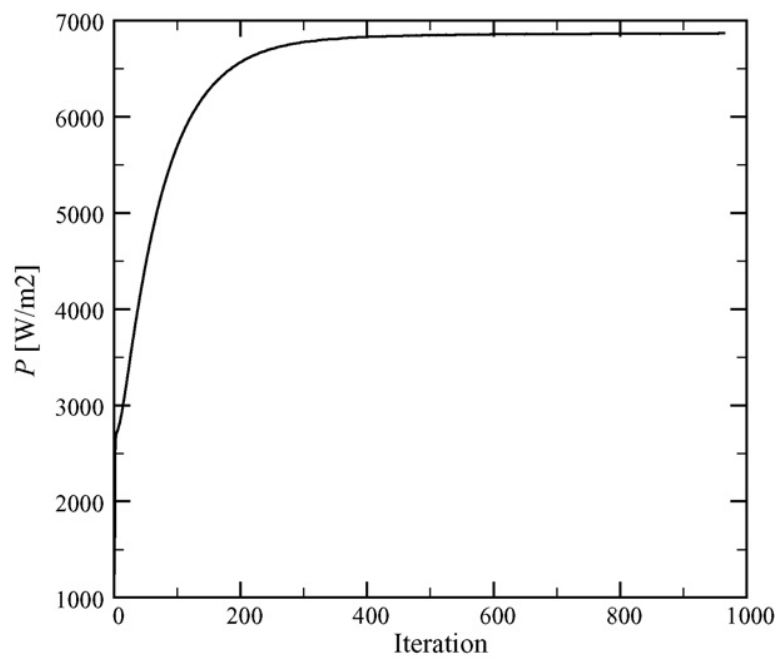

Fig. 8. Variation in power output during the optimization process.

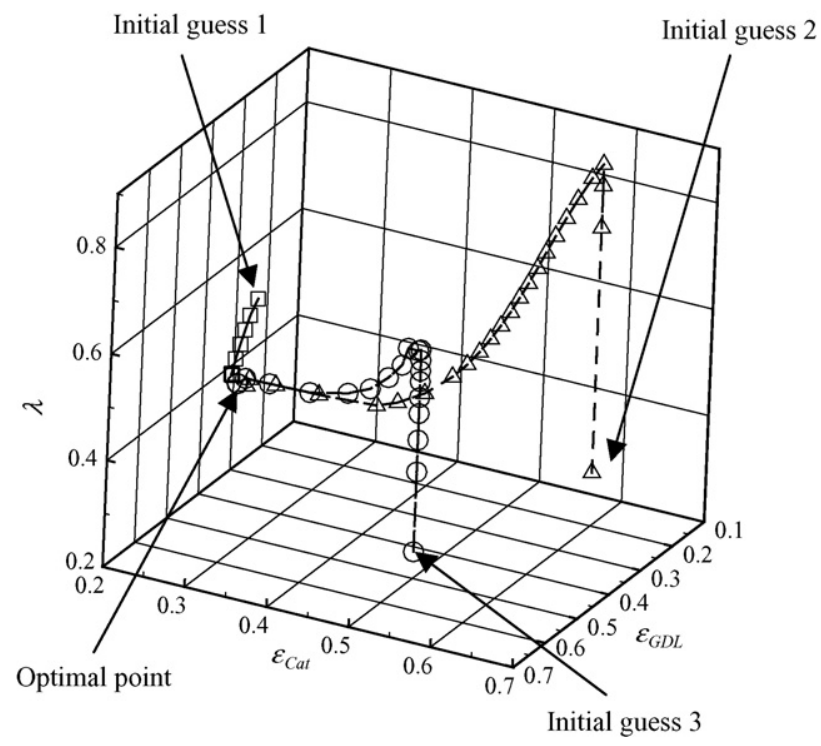

Fig. 9. Trajectories of the optimization processes from different sets of initial guesses for $\lambda, \varepsilon_{\mathrm{GDL}}$, and $\varepsilon_{\mathrm{Cat}}$

that the present optimization method leads to the same optimal set of parameters with different initial guesses.

\section{Conclusions}

In this study, an optimization analysis employing the SCGM method [10] combined with a two-dimensional half-cell model for the transport phenomena has been successfully developed. The optimization of design parameters $\lambda, \varepsilon_{\mathrm{GDL}}, \varepsilon_{\mathrm{Cat}}$, has been performed. The results show that, at the conditions of $\lambda=0.55$, $\varepsilon_{\mathrm{GDL}}=0.5913$ and $\varepsilon_{\mathrm{Cat}}=0.307$, the optimal cell performance in terms of power is $P=6866.53 \mathrm{~W} \mathrm{~m}^{-2}$. Extension of the present method to one with more parameters is a worthwhile work for the future, and a more comprehensive model would also be interesting. It is noted that the present optimization approach is not limited to the present group of designed parameters. Where necessary, more designed parameters may be readily applied.

\section{References}

[1] P.L. Hental, J.B. Lakeman, G.O. Mesped, P.L. Adock, J.M. Moor, New materials for polymer electrolyte membrane fuel cell current collectors, J. Power Sources 80 (1999) 235-241.

[2] E. Hontañón, M.J. Escudero, C. Bautista, P.L. García-Ybarra, L. Daza, Optimization of flow-field in polymer electrolyte membrane fuel cells using computational fluid dynamics techniques, J. Power Sources 86 (2000) 363-368.

[3] Y.G. Yoon, W.Y. Lee, G.G. Park, T.H. Yang, C.S. Kim, Effects of channel configurations of flow field plates on the performance of a PEMFC, Electrochim. Acta 50 (2004) 709-712.

[4] V. Gurau, H. Liu, S. Kakac, Two-dimensional model for proton exchange membrane fuel cells, AICHE J. 137 (11) (1998) 2410-2422.

[5] J.T. Wang, R.F. Savinell, Simulation studies on the fuel electrode of a $\mathrm{H}_{2}-\mathrm{O}_{2}$ polymer electrolyte fuel cell, Electrochim. Acta 37 (1992) 2737-2745.

[6] W.M. Yan, C.Y. Soong, F.L. Chen, H.S. Chu, Effects of flow distributor geometry and diffusion layer porosity on reactant gas transport and performance of proton exchange membrane fuel cells, J. Power Sources 125 (2004) 27-39. 
[7] T. Zhou, H. Liu, A 3D model for PEM fuel cells operated on reformate, J. Power Source 138 (2004) 101-110.

[8] A. Kumar, R.G. Reddy, Effect of channel dimensions and sharp in the flowfield distributor on the performance of polymer electrolyte membrane fuel cell, J. Power Sources 113 (2003) 11-18.

[9] H.C. Hsu, Study of transport phenomena and optimal design for proton exchange membrane fuel cells, Master of Science (Advisor: C. H Cheng), Department of Mechanical Engineering, Tatung University, Taipei, Taiwan, 2004.

[10] W. Ying, T.H. Yang, W.Y. Lee, J. Ke, C.S. Kim, Three-dimensional analysis for effect of channel configuration on the performance of a small airbreathing proton exchange membrane fuel cell (PEMFC), J. Power Sources 145 (2005) 572-581.

[11] K.W. Lum, J.J. McGuirk, Three-dimensional model of a complete polymer electrolyte membrane fuel cell-model formulation, validation and parametric studies, J. Power Source 143 (2005) 103-124.

[12] T. Berning, N. Djilali, Three-dimensional computational analysis of transport phenomena in a PEM fuel cell-a parametric study, J. Power Source 124 (2003) 440-452.
[13] I. Mohamed, N. Jenkins, Proton exchange membrane (PEM) fuel cell stack configuration using genetic algorithms, J. Power Source 131 (2004) 142-146.

[14] C.H. Cheng, M.H. Chang, A simplified conjugate-gradient method for shape identification based on thermal data, Numer. Heat Transfer, Part B 43 (2003) 489-507.

[15] Y. Hung, N.G. Zamani, Optimum beam design using finite elements, Appl. Math. Comput. 80 (1996) 155-179.

[16] P.C. Tuan, M.C. Ju, The validation of the robust input estimation approach to two-dimensional inverse heat conduction problems, Numer. Heat Transfer, Part B 37 (2000) 247-265.

[17] C.H. Cheng, M.H. Chang, Predictions of internal temperature distribution of PEMFC by non-destructive inverse method, J. Power Source 139 (2005) $115-125$.

[18] M.H. Chang, C.H. Cheng, Non-destructive inverse method for determination of irregular internal temperature distribution in PEMFCs, J. Power Source 142 (2005) 200-210. 